\title{
Coupling of quantum gravitational field with Riemann and Ricci curvature tensors
}

\author{
Claudio Cremaschini ${ }^{1, \mathrm{a}}$, Massimo Tessarotto ${ }^{1,2, \mathrm{~b}}$ \\ ${ }^{1}$ Research Center for Theoretical Physics and Astrophysics, Institute of Physics, Silesian University in Opava, Bezručovo nám.13, 74601 Opava, \\ Czech Republic \\ ${ }^{2}$ Department of Mathematics and Geosciences, University of Trieste, Via Valerio 12, 34127 Trieste, Italy
}

Received: 15 May 2021 / Accepted: 15 June 2021 / Published online: 25 June 2021

(C) The Author(s) 2021

\begin{abstract}
The theoretical problem of establishing the coupling properties existing between the classical and quantum gravitational field with the Ricci and Riemann curvature tensors of General Relativity is addressed. The mathematical framework is provided by synchronous Hamilton variational principles and the validity of classical and quantum canonical Hamiltonian structures for the gravitational field dynamics. It is shown that, for the classical variational theory, manifestly-covariant Hamiltonian functions expressed by either the Ricci or Riemann tensors are both admitted, which yield the correct form of Einstein field equations. On the other hand, the corresponding realization of manifestlycovariant quantum gravity theories is not equivalent. The requirement imposed is that the Hamiltonian potential should represent a positive-definite quadratic form when performing a quadratic expansion around the equilibrium solution. This condition in fact warrants the existence of positive eigenvalues of the quantum Hamiltonian in the harmonic-oscillator representation, to be related to the graviton mass. Accordingly, it is shown that in the background of the deSitter spacetime, only the Ricci tensor coupling is physically admitted. In contrast, the coupling of quantum gravitational field with the Riemann tensor generally prevents the possibility of achieving a Hamiltonian potential appropriate for the implementation of the quantum harmonic-oscillator solution.
\end{abstract}

\section{Introduction}

This paper deals with a conceptual issue that lays at the basis of the foundations of quantum gravity $(\mathrm{QG})$ theory, and specifically the manifestly-covariant quantum gravity theory (CQG-theory), i.e., the massive-graviton and 4-tensor theory of manifestly-covariant QG which pertains the Einstein

\footnotetext{
a e-mail: claudiocremaschini@gmail.com (corresponding author)

be-mail: maxtextss@gmail.com
}

Field Equations (EFE) and the related abstract 4-tensor classical Hamiltonian structure of General Relativity (GR) [1,2]. This concerns the coupling properties of the quantum gravitational field with curvature tensors of the background spacetime. The latter are identified respectively with the 4-rank Riemann curvature tensor $R_{\mu \alpha \nu \beta}$ and the associated 2-rank Ricci tensor $R_{\mu \nu}$. In particular, the question addressed here is whether the physical interaction of the quantum field with the two tensors $R_{\mu \alpha \nu \beta}$ and $R_{\mu \nu}$ exhibits the same qualitative properties or if it corresponds to different quantum-gravity dynamical realizations. The proper understanding of the matter is crucial because it permits us to unveil also the possible quantum implications of such a coupling on the construction of the global solution for the space-time metric tensor [3-8]. The issue is not a trivial one. In fact, the role of the two curvature tensors, as we intend to show here, is not equivalent. Apart their rank, the Riemann tensor is a fundamental curvature tensor of differential geometry, while the symmetric Ricci curvature tensor is derived by means of a suitable "tensor coupling", i.e., by suitable tensor index saturation of the Riemann tensor with a metric tensor [9]. The latter tensor, however, in principle is not necessarily the extremal one, i.e., the background metric tensor solution of the classical field equations, but can exhibit certain 'ad hoc' properties (i.e., for example being identified with an appropriate variational metric tensor). Thus, specifically for this reason, a priori one is not allowed to assume that the coupling occurs in the same way for the two cases. As a consequence, it is not possible to rule out the possibility that there exist fundamental physical reasons for selecting a specific type of tensor coupling and in turn the outcome on physical observables.

The problem becomes even more significant when quantum gravity theory is concerned. In addition, in the same framework there can be a quantum-indeterminacy character to be attributed to Riemann and Ricci tensors. This depends on whether they are regarded as purely classical background 
and possibly prescribed continuum tensors or if they possess by themselves a quantum nature and how this can emerge consistently. Therefore, the nature of the problem pertains intrinsically both classical and quantum gravity theories [10 12].

In this regard it is useful to recall what happens in the case of the Einstein-Hilbert (EH) Lagrangian variational approach (referred to for brevity as EH theory [13]), originally developed by Einstein himself to determine the actual form of GR field equations. As shown in Ref. [14], EH-theory relies on the adoption of an asynchronous variational principle. The latter is based on a Lagrangian density of the type $L_{E H}=\sqrt{-g} R$ (with $g$ denoting as usual the determinant of the metric tensor, i.e., $g \equiv|g|)$ and $R$ is the Ricci 4-scalar, which in terms of the so-called Ricci-tensor coupling, reads

$R=g^{\mu v} R_{\mu \nu}$.

This means that in the framework of asynchronous principles the variation of the invariant volume element $d \Omega=d^{4} r \sqrt{-g}$ yields non-vanishing contributions, while correspondingly the variational Lagrangian density is not a 4-scalar, namely a frame-independent quantity. Because the variational contribution of $\sqrt{-g}$ carried by $d \Omega$ is uniquely prescribed, $\mathrm{EH}$ theory demands the aforementioned tensor coupling to be prescribed in terms of the Ricci tensor, i.e., Eq. (1). In other words, the Ricci 4-scalar in the previous functional must necessarily be expressed as saturation of the symmetric Ricci 4-tensor in order to warrant the variational nature of the Lagrangian density. The fundamental reason why in EHtheory the same coupling is a privileged one is very simple. As shown in the same reference indicated above, EFE are formally obtained by requiring that the variational contribution to the corresponding Euler-Lagrange equations carried by the variational Ricci tensor, i.e., the contributions carried by its Frechet derivatives $\delta R_{\mu \nu}$ and $\delta R^{\mu \nu}$, should vanish identically.

Nevertheless, even if in the context of EH-theory a Riemann-tensor variational formulation of EFE is not extant, it is obvious that at the classical level the issue of the possible role of the Riemann-tensor coupling arises also in the framework of the asynchronous variational theory of GR. In fact, although the tensor coupling (1) is commonly regarded as the simplest and the logically well-founded one for the gravitational-field Lagrangian density [9], in principle a possible alternative exists. This is based on the Riemann-tensor tensor coupling mentioned above, being represented by the equation

$R=g^{\mu \nu} g^{\alpha \beta} R_{\mu \alpha \nu \beta}$.

Such a tensor coupling yields, however, via the EinsteinHilbert action principle, Euler-Lagrange equations which are unavoidably different from EFE. A detailed derivation of such equations, based on the EH asynchronous variational principle, can be found in Ref. [15]. This kind of analysis is, however, outside the scope of the present investigation, which concerns primarily EFE and its quantum theory.

However, the adoption of a synchronous variational principle, in place of the asynchronous one, changes matter (see Ref. [16]). The synchronous variational approach in fact is characterized by the use of a 4-scalar variational Lagrangian function expressed in terms of superabundant variables, namely in which the variational metric tensor is distinguished from the prescribed background one, to be ultimately determined consistently by the extremal EulerLagrange equations. Thus, the invariant volume element $d \Omega$ is represented in terms of a suitably-prescribed, i.e., extremal and hence non variational, metric tensor $\widehat{g} \equiv\left\{\widehat{g}_{\mu \nu}\right\}$, namely defined so that its Frechet derivative $\delta \widehat{g}$ vanishes identically. Thus, letting $d \Omega=d^{4} r \sqrt{-\widehat{g}}$, with $\widehat{g} \equiv|\widehat{g}|$ now denoting the determinant of $\widehat{g}$, it follows that $d \Omega$ does not contribute to the variational calculus since by construction $\delta d \Omega=0$. From the conceptual point of view, this kind of approach has similarities with the theoretical formulation of GR based on the use of a metric independent volume element, which is known as the Non-Gravitating Vacuum Energy Theory [17-19]. The latter is based on replacing $\sqrt{-g}$ by a non-Riemannian measure density $\Phi$ for the volume element which does not depend on the metric and is at the same time a total divergence. This kind of formulation is implemented in the framework of a non-Riemannian geometry, while validity of the standard formulation of GR with Riemannian geometry is assumed for the synchronous principle proposed in Refs. $[1,16]$. The same synchronous variational principle adopts the customary identification of $R$ in terms of the Ricci 4-tensor, a choice inherited also by the related quantum Hamiltonian potential. However, the advantage of the synchronous approach over the asynchronous one is that the Lagrangian density is independent of the factor $\sqrt{-g}$ and is therefore a 4-scalar. This provides in principle the required freedom to investigate also the variational role of the Riemann tensor in comparison with the Ricci one. In fact, in such a framework, one can investigate alternative expressions of the Lagrangian so that it contains the Riemann tensor, circumventing the constraint placed by the variation of $d \Omega$ which would provide instead always the same contribution, independent of the precise realization of the Lagrangian function.

Therefore, the synchronous setting and its realization in terms of manifestly-covariant classical and quantum gravity theories (CCG- and CQG- theories) provide the required freedom to reach the target. More precisely, given these premises, the main goals of the paper are as follows:

(1) We first prove that adopting the synchronous approach it is possible to construct two distinctive, but equivalent, classical variational principles for EFE which are expressed respectively in terms of either the Ricci or Riemann-tensor 
couplings, and in which the Ricci or the Riemann tensors are considered extremal. Remarkably both variational principles are found to recover correctly the same form of EFE in terms of their extremal Euler-Lagrange equations. It is shown, however, that - apart arbitrary gauge functions - the prescription of the variational Hamiltonian represented in terms of the Riemann-tensor coupling remains not unique. Nevertheless, by suitable adoption of an appropriate physical criterion, the selection of a unique physically-meaningful form is shown to be possible. The criterion states the requirement that the variational functional expressed via the Riemann tensor must recover a posteriori the customary one containing the Ricci tensor when the relationship between the Ricci and the Riemann tensors is invoked. It is important to stress, however, that such criterion is not mandatory by any means. The conclusion it therefore that at classical level both variational principles, i.e., expressed in terms of either the Ricci or Riemann tensors, are in principle acceptable, as far as they yield the same correct form of EFE among the extremal equations.

(2) Second, we show that the selection of the appropriate variational approach can (only) be established in the framework of quantum theory. To do so, we consider a background space-time realized by the vacuum deSitter solution generated by a non-vanishing cosmological constant (CC). In the context of CQG-theory, considered here as a reference theory, the presence of a non-vanishing (and strictly positive) CC is, in fact, a mandatory consequence of the Bohm vacuum quantum interaction occurring among gravitons, as predicted by the same theory [20]. The validity of CQG-theory requires however the existence of massive gravitons. The fact that the gravitons should be massive implies, on the other hand, the requirement that the quantum (4-scalar) Hamiltonian must necessarily admit a universal harmonic oscillator form, i.e., positive-definite quadratic form, and a consequent spectrum of discrete positive eigenvalues (determining the spectrum of possible invariant rest masses for the gravitons) in the presence of a non-vanishing Hamiltonian potential. Under these circumstances, it is found that the realization of the Lagrangian density is unique. In particular, this means that the Riemann-tensor coupling must be ruled out, because it violates the requirement of realizing a quadratic and positivedefinite harmonic Hamiltonian potential. It is proved that this deficiency which characterizes the Riemann-tensor solution with respect to the Ricci-tensor one is ultimately due to the coupling between the quantum gravitational field and the same non-symmetric Riemann tensor of the deSitter spacetime. This permits to conclude that the quantum gravitational field (and in particular its stationary solution with discrete eigenvalues) selects the coupling with the Ricci tensor. The conclusion excludes the role of the Riemann tensor in CQGtheory having a deSitter background solution, and in turn it gives support to the same CQG formalism developed so far.

\section{Physical requirements and mathematical setting}

The question of the quantum coupling must be set in the appropriate mathematical framework. This is identified with the classical variational theory of GR for the continuum gravitational field and the corresponding canonical formulation of quantum gravity theory. More precisely, the nature of the problem demands to satisfy the following requirements:

1. The principle of manifest covariance (PMC). This states that it should always be possible to cast the physical laws of relativistic field theories in 4-tensor form with respect to the group of local point transformations which leaves invariant the differential manifold of the background space-time [13]. The validity of such a principle provides strict guidelines on the formal representation of mathematical relationships holding among the physical laws. It provides constraints on the admissible realization (and possible universal character) of the relevant equations, physical parameters, classical or quantum phasefunctions and observables. In this sense, PMC affects both the classical and quantum descriptions of continuum fields in relativistic field theories, for the search respectively of appropriate variational formulations for classical field theories and of canonical quantization methods [21].

2. The validity of a classical Hamiltonian theory of GR consistent with PMC. This is realized by identifying an invariant evolution parameter $s$, to be denoted proper-time, and introducing the set $\{x, H\}$, formed by the canonical state $x(s) \equiv(g(s), \pi(s))$ and a suitable classical Hamiltonian density $H$. Here, $g(s)=\left\{g_{\mu \nu}(s)\right\}$ and $\pi(s)=\left\{\pi^{\mu \nu}(s)\right\}$ denote respectively the variational tensor field and the conjugate tensor canonical momentum. The canonical state $x(s)$ must therefore fulfill a corresponding set of continuum Hamilton equations

$\left\{\begin{array}{l}\frac{d g_{\mu v}}{d s_{v}}=\left[g_{\mu \nu}, H\right]=\frac{\partial H}{\partial \pi^{\mu \nu}}, \\ \frac{d \pi^{\mu \nu}}{d s}=\left[\pi^{\mu \nu}, H\right]=-\frac{\partial H}{\partial g_{\mu v}},\end{array}\right.$

subject to the initial-value condition

$x\left(s_{o}\right) \equiv\left(g_{\mu \nu}\left(s_{o}\right), \pi^{\mu \nu}\left(s_{o}\right)\right)$.

Here, $g_{\mu \nu}\left(s_{o}\right)$ and $\pi^{\mu \nu}\left(s_{o}\right)$ denote two initial conjugate tensor fields, $s_{o}$ is the initial proper-time and $\frac{d}{d s}$ is a suitable covariant $s$-derivative operator to be properly defined [1]. Then, the solution of the initial-value problem (3)-(4) generates the Hamiltonian flow

$x\left(s_{o}\right) \rightarrow x(s)$,

which is associated with the Hamiltonian structure $\{x, H\}$. For the validity of the theory, the canonical equations 
(3) must recover correctly the form of EFE among their extremal solutions, so that $\{x, H\}$ effectively identifies a Hamiltonian structure of GR.

3. The validity of a quantum Hamiltonian theory of GR. Given the classical GR-Hamiltonian structure, this is represented by the set $\left\{x^{(q)}, H^{(q)}\right\}$ realized by the formal map

$$
\left\{\begin{array}{c}
g_{\mu \nu} \rightarrow g_{\mu \nu}^{(q)} \equiv g_{\mu \nu}, \\
\pi_{\mu \nu} \rightarrow \pi_{\mu \nu}^{(q)} \equiv-i \hbar \frac{\partial}{\partial g^{\mu \nu}}, \\
H \rightarrow H^{(q)},
\end{array}\right.
$$

where the dimensional 4-scalar $H^{(q)}$ identifies the quantum Hamiltonian operator, $x^{(q)} \equiv\left\{g_{\mu \nu}^{(q)}, \pi_{\mu \nu}^{(q)}\right\}$ is the quantum canonical state and $\pi_{\mu \nu}^{(q)}$ is the quantum momentum operator prescribed so that the commutator $\left[g_{\mu \nu}^{(q)}, \pi^{(q) \alpha \beta}\right]=i \hbar \delta_{\mu}^{\alpha} \delta_{\nu}^{\beta}$ applies. It is understood that the actual realization of the mapping (6) requires also the prescription of dimensional constants to warrant the correct physical dimensions of the quantum Hamiltonian function and quantum operators (see Ref. [2] and Sect. 5 below). Such a quantum correspondence must then imply the realization of a manifestly-covariant Schrödinger-like quantum-wave equation for a quantum wave function $\psi$ of the form

$i \hbar \frac{\partial}{\partial s} \psi(s)=\left[H^{(q)}, \psi(s)\right] \equiv H^{(q)} \psi(s)$,

with $\frac{\partial}{\partial s}$ denoting a covariant $s$-derivative and $[A, B]$ being the quantum commutator $[A, B] \equiv A B-B A$. In the present setting, the quantum wave function $\psi$ must admit a Madelung representation

$\psi(s)=\sqrt{\rho(s)} \exp \left\{\frac{i}{\hbar} S^{(q)}(s)\right\}$,

where the quantum fluid fields $\left\{\rho(s), S^{(q)}(s)\right\}$ identify respectively the quantum probability density function (PDF) and the quantum phase-function. As a consequence, the acceptable quantum gravity theory must preserve the probabilistic interpretation characteristic of quantum mechanics, and in particular it must warrant validity of the Born rule, so that the 4-scalar $\rho(s) \equiv$ $|\psi(s)|^{2}$ is a probability density on the 4-dimensional space-time.

The requirement of consistency with prescriptions 1-3 selects the admissible type of framework for the investigation of the problem posed here. In particular, approaches based on non-manifestly covariant and non-Hamiltonian theories like ADM-formulation and the Wheeler-deWitt equa- tion rooted on Loop Quantum Gravity remain excluded [2224] (see also discussion in Ref. [14]). The appropriate setup which realizes the scheme proposed above is provided by the manifestly-covariant classical and quantum theories of the gravitational field (denoted in short-way respectively as CCG- and CQG- theories). These are formulated based on the validity of synchronous Lagrangian and Hamiltonian variational principles. Characteristic features of the synchronous approach with respect to the customary asynchronous principles widespread in the literature are the space-time representation in terms of superabundant field variables $(\widehat{g}, g)$ and the metric tensor background formalism. Accordingly, manifest covariance is defined with respect to a continuum background metric tensor $\widehat{g} \equiv\left\{\widehat{g}_{\mu \nu}\right\}$, which raises/lowers tensor indices and defines the geometric properties of the spacetime, including its volume element. The same background metric tensor is then determined self-consistently as a solution of quantum-modified Einstein field equations implied by the same CQG quantum-wave equation. In contrast, the variational theory applies to the tensor field $g \equiv\left\{g_{\mu \nu}\right\}$, which has generally a non-vanishing covariant derivative in the action functional, so that $\widehat{\nabla}_{\alpha} g^{\mu \nu} \neq 0$, where $\widehat{\nabla}_{\alpha}$ is the covariant derivative whose connections are expressed in terms of $\widehat{g}_{\mu \nu}$. The field $g_{\mu \nu}$ is ultimately identified with the quantum gravitational field of the quantum Hamiltonian theory. As such, the quantum field $g_{\mu \nu}$ can evolve dynamically over $\widehat{g}$ according to the quantum wave equation (CQG-wave equation), so that it can acquire correspondingly a non-vanishing quantum momentum $\pi_{\mu \nu}$. By definition the tensor $g_{\mu \nu}$ is such that $g^{\mu \nu} g_{\mu \nu} \neq \delta_{\mu}^{\mu}$, while the normalization condition $\widehat{g}^{\mu \nu} \widehat{g}_{\mu \nu}=4$ applies to the classical metric tensor. A main result to be mentioned here is the proof that CQG-theory predicts the existence of a discrete eigenvalue spectrum for the quantum gravitational field. Its analytical treatment is permitted by the formalism of manifest covariance and the implementation of the Dirac ladder method [25]. Thus, in vacuum configurations and provided there exists a non-vanishing cosmological constant generating a curvature potential, the eigenvalue spectrum recovers also in such a context the fundamental harmonic-oscillator solution [2].

The detailed derivation and the physical implications of CCG- and CQG- theories is discussed in the following sections, for both Ricci and Riemann tensor coupling solutions.

\section{Classical Hamilton variational principle: the Ricci tensor}

We recall the synchronous classical Hamilton variational principle underlying EFE expressed in terms of the Ricci tensor. Following the derivation reported in Ref. [1], we denote by $x(s) \equiv(g(s), \pi(s))$ the variational canonical state expressed in terms of the generalized coordinate $g(s)=$ 
$g_{\mu \nu}(s)$ and its conjugate reduced momentum $\pi(s)=$ $\pi^{\mu \nu}(s)$, and by $\widehat{x}(s) \equiv(\widehat{g}(s), \widehat{\pi}(s))$ the corresponding prescribed state. Then, in such a case the Hamiltonian density $H$ is identified with $H \equiv H_{h}$, with $H_{h} \equiv H_{h}(x, \widehat{x}, r, s)$ being expressed by the 4-scalar

$H_{h}=T-V_{h}$,

where the effective kinetic and potential densities $T \equiv$ $T(x, \widehat{x})$ and $V_{h} \equiv V_{h}(g, \widehat{x}, r, s)$ are taken respectively of the form

$$
\begin{aligned}
T(x, \widehat{x}) & \equiv \frac{1}{2 \kappa} \pi_{\mu \nu} \pi^{\mu \nu}, \\
V_{h}(g, \widehat{x}, r, s) & \equiv V_{G h}(g, \widehat{x})+V_{F}(g, \widehat{x}, r, s) .
\end{aligned}
$$

Here $\kappa$ is the dimensional constant $\kappa=\frac{c^{3}}{16 \pi G}$, while by construction $\pi^{\mu \nu}=\kappa t^{\alpha} \widehat{\nabla}_{\alpha} g^{\mu \nu}$, where $t^{\alpha}$ is the dimensionless unit 4-vector tangent to a subliminal geodesic trajectory of $\widehat{g}_{\mu \nu}$. Furthermore, the two contributions in the potential density are expressed as

$$
\begin{aligned}
V_{G h}(g, \widehat{x}) & \equiv \kappa h\left[g^{\mu \nu} \widehat{R}_{\mu \nu}-2 \Lambda\right], \\
V_{F}(g, \widehat{x}, r) & \equiv h L_{F}(g, \widehat{x}, r),
\end{aligned}
$$

where $V_{G h}(g, \widehat{x})$ is the gravitational part of the potential, while $V_{F}(g, \widehat{x}, r)$ identifies the external-field source contributions, with $L_{F}$ being associated with a non-vanishing stress-energy tensor due to external non-gravitational fields. We notice in particular that the potential $V_{G h}$ contains the Ricci tensor $\widehat{R}_{\mu \nu}$, which in the framework of synchronous variational principle is assumed as prescribed (i.e., it is non variational). In addition, for completeness we have included the contribution of a non-vanishing cosmological constant $\Lambda$. The variational weight-factor $h=h(g, \widehat{x}, r)$, which appears as a common factor in the potential part of the Hamiltonian, $V_{h}(g, \widehat{x}, r, s)$, has an important role. According to Ref. [16], in fact, it is found that for the correct determination of EFE the variational weight-factor $h$ must be identified with the function

$h(g, \widehat{x}, r)=\left(2-\frac{1}{4} g^{i k} g_{i k}\right)$,

and hence prescribed so that

$h(\widehat{g}, \widehat{x}, r)=1$.

We stress that the choice of the variational weight-factor $h$ given here can be shown to be unique, i.e., provided it is identified with a second-degree polynomial in $g_{i k}$. It is worth recalling briefly here the physical meaning of the function $h$ [16]. This quantity in fact marks the connection between the synchronous functional and the asynchronous one. More precisely, in the synchronous variational principle, $h$ replaces the variational contribution of the metric tensor which in the asynchronous principle originates from the variation of $\sqrt{-g}$. In fact, $h$ is constructed in such a way that

$\delta h(g, \widehat{x}, r)=\delta \sqrt{-g}$.

There are a few crucial points to stress here. First, although $h$ and $\sqrt{-g}$ yield the same variations, they have a different tensor character. In fact, while $\sqrt{-g}$ is obviously not a 4scalar quantity, the function $h$ realizes, instead, by construction a 4-scalar. Second, as stated in Eq. (13) above, we also notice that the function $h$ equally applies also to external-field Lagrangians. Hence, it follows that the replacement of $\sqrt{-g}$ with $h$ holds consistently both in vacuum and non-vacuum cases. The crucial implication of these two features is that the Hamiltonian density $H$ acquires indeed a 4-scalar character.

Such conditions realize therefore also a mandatory requirement for achieving a manifestly covariant variational principle which is realized by the synchronous Hamilton variational principle in terms of the 4-scalar variational functional

$S_{h}(x, \widehat{x}) \equiv \int d \Omega L_{h}(x, \widehat{x}, r, s)$

with $d \Omega$ identifying the invariant 4-volume element of the space-time. In addition, $L_{h}(x, \widehat{x}, r, s)$ is the variational Lagrangian density

$L_{h}(x, \widehat{x}, r, s) \equiv \pi_{\mu \nu} \frac{d}{d s} g^{\mu \nu}-H_{h}(x, \widehat{x}, r, s)$,

to be identified with the Legendre transform of the corresponding variational Hamiltonian density $H_{h}(x, \widehat{x}, r, s)$ defined above, with $\pi_{\mu \nu} \frac{d}{d s} g^{\mu \nu}$ denoting the so-called exchange term. Here, $\frac{d}{d s}$ is the covariant $s$-derivative

$\frac{d}{d s}=\frac{\partial}{\partial s}+t^{\alpha}(s) \widehat{\nabla}_{\alpha}$

while $t^{\alpha}(s)$ and $\widehat{\nabla}_{\alpha}$ are respectively the tangent 4 -vector to the geodesics trajectory $r(s) \equiv\left\{r^{\mu}(s)\right\}$ and the covariant derivative evaluated in terms of the prescribed metric tensor $\widehat{g}_{\mu \nu}$. Then, the variational principle associated with the functional $S_{h}(x, \widehat{x})$ is prescribed in terms of the synchronousvariation operator $\delta$, to be identified with the Frechet derivative according to Ref. [16]. According to such an approach the 4-scalar $d \Omega$ entering the action functional is considered independent of the functional class of variations, so that it must be defined in terms of the prescribed metric tensor field $\widehat{g}$ as $d \Omega \equiv d^{4} r \sqrt{-|\widehat{g}|}$, with $d^{4} r$ being the corresponding canonical measure and $|\widehat{g}|$ denoting as usual the determinant of the metric tensor $\widehat{g}(r)$. In the context of the synchronous variational principle the prescribed field $\widehat{g}_{\mu \nu}(r)$ must determine, besides $d \Omega$, also the geometric properties of space-time. This 
requires in particular that $\widehat{g}_{\mu \nu}(r)$ must lower and raise tensor indexes of tensor fields and determine also the standard Christoffel connections which enter both the Ricci tensor $\widehat{R}_{\mu \nu}$ and the covariant derivatives of arbitrary variational tensor fields. Therefore, the synchronous variational principle to GR realizes the so-called "background space-time picture", whereby the background space-time $\left(\mathbf{Q}^{4}, \widehat{g}(r)\right)$ is considered defined "a priori" in terms of $\widehat{g}_{\mu \nu}(r)$, while leaving unconstrained all the variational fields $x=\{g, \pi\}$ and in particular the Lagrangian coordinates $g(r) \equiv\left\{g_{\mu \nu}(r)\right\}$. Thus, denoting

$\delta S_{h}(x, \widehat{x})=\left.\frac{d}{d \lambda} S_{h}(x+\lambda \delta x, \widehat{x})\right|_{\lambda=0}$

the Frechet derivative of the functional $S_{h}(x, \widehat{x})$, the corresponding synchronous variational principle is realized by means of the variational equation

$\delta S_{h}(x, \widehat{x})=0$,

namely obtained by keeping constant both the prescribed state $\widehat{x}$ and the 4 -scalar volume element $d \Omega$. This delivers the 4-tensor Euler-Lagrange equations cast in symbolic form in terms of the variational derivatives $\frac{\delta S_{h}(x, \widehat{x})}{\delta g^{\mu \nu}}$ and $\frac{\delta S_{h}(x, \widehat{x})}{\delta \pi_{\mu \nu}}$

$\left\{\begin{array}{l}\frac{\delta S_{h}(x, \widehat{x})}{\delta g^{\mu \nu}}=0, \\ \frac{\delta S_{h}(x, \widehat{x})}{\delta \pi_{\mu \nu}}=0,\end{array}\right.$

which are equivalent to the Hamilton equations (3) upon letting $H=H_{h}$.

In particular, we have that

$\frac{\delta\left[h g^{\mu \nu} \widehat{R}_{\mu \nu}\right]}{\delta g^{\mu \nu}}=h \widehat{R}_{\mu \nu}-\frac{1}{2} g_{\mu \nu}\left(g^{l m} \widehat{R}_{l m}\right)$,

so that invoking Eqs. (11)-(13) it follows that

$$
\begin{aligned}
& \frac{\partial V_{G h}(g, \widehat{x}, r, s)}{\partial g^{\mu \nu}(s)}=\kappa h(s) \widehat{R}_{\mu \nu} \\
& -\kappa g_{\mu \nu}(s) \frac{1}{2}\left(g^{l m}(s) \widehat{R}_{l m}-2 \Lambda\right)-\kappa \frac{8 \pi G}{c^{2}} T_{\mu \nu},
\end{aligned}
$$

where $\widehat{R}_{\mu \nu} \equiv \widehat{R}_{\mu \nu}(s)$ and $T_{\mu \nu} \equiv T_{\mu \nu}(s)$ denote the Ricci and stress-energy tensors. Then, the canonical equations (3) reduce to the single equivalent Lagrangian evolution equation for the variational field $g_{\mu \nu}(s)$ given by

$$
\begin{aligned}
\frac{d}{d s} & {\left[\frac{d}{d s} g_{\mu \nu}(s)\right]+h(s) \widehat{R}_{\mu \nu}-g_{\mu \nu}(s) } \\
& \frac{1}{2}\left[g^{l m}(s) \widehat{R}_{l m}-2 \Lambda\right]-\frac{8 \pi G}{c^{2}} T_{\mu \nu}=0 .
\end{aligned}
$$

The connection of the canonical equations (3), and therefore of Eq. (25), with EFE can be obtained under the assumption that the Hamiltonian density does not depend explicitly on proper time $s$, i.e., it is actually of the form

$H_{h}=H_{h}(x, \widehat{x}(r), r)$,

and by imposing the stationary initial conditions on the state $x\left(s_{o}\right)$ of the type

$\left\{\begin{array}{l}g_{\mu \nu}\left(s_{o}\right) \equiv \widehat{g}_{\mu \nu}\left(s_{o}\right), \\ \pi_{\mu \nu}\left(s_{o}\right) \equiv \widehat{\pi}_{\mu \nu}\left(s_{o}\right)=0,\end{array}\right.$

while requiring furthermore for all $s \in I$

$\widehat{\pi}_{\mu \nu}(s)=0$.

Then, since the identities $\widehat{g}_{\mu \nu}(s) \widehat{g}^{\mu \nu}(s)=\delta_{\mu}^{\mu}$ and $\frac{d}{d s} \widehat{g}_{\mu \nu}(s) \equiv$ 0 hold by construction, so that necessarily $\widehat{\pi}_{\mu \nu}(s) \equiv 0$, Eq. (25) reduces to

$\widehat{R}_{\mu \nu}-\widehat{g}_{\mu \nu}(s) \frac{1}{2}\left[\widehat{g}^{l m}(s) \widehat{R}_{l m}-2 \Lambda\right]=\frac{8 \pi G}{c^{2}} \widehat{T}_{\mu \nu}$,

which coincides with the Einstein field equations solving for the prescribed field $\widehat{g}_{\mu \nu}$.

As a final remark, we stress that the presence of the function $h$ is a unique characteristic feature of the synchronous variational principle with respect to the analogous asynchronous problem. From the physical point of view, the function $h$ replaces the variational contributions arising from variations of the volume element $d \Omega$. In the framework of a background space-time formalism and adoption of superabundant coordinate field variables, the function $h$ can also be interpreted as measuring the deviation of the variational field $g_{\mu \nu}$ with respect to $\widehat{g}_{\mu \nu}$. In fact, the extremal value of $h$ is identically 1 , since in this limit $g$ coincides with $\widehat{g}$. We also recall that the adoption of the synchronous principle together with the inclusion of the 4-scalar function $h$ restores the correct invariance gauge properties of the Lagrangian function, and therefore of the Hamiltonian function too. In particular, in the present case $H_{h}$ is found to satisfy the principle of manifest covariance, establishing a variational principle which does not rely anymore on the variation of the non-covariant contribution $\sqrt{-|g|}$ defining the volume element of the action functional.

\section{Classical Hamilton variational principle: the Riemann tensor}

In this section we investigate the possibility of formulating a classical Hamilton variational principle such that the corresponding Hamiltonian potential is expressed in terms of the 
Riemann tensor instead of the Ricci tensor. The same principle must be consistent with the principle of manifest covariance and it must recover the correct form of EFE among its extremal equations. We intend to prove that the task can be consistently realized in the framework of synchronous variational principles. More precisely, we show that the solution for the Hamiltonian potential is generally non-unique. However, by prescription of suitable physical requisites the appropriate form of the Hamiltonian function is selected and its relationship with $H_{h}$ obtained above can be displayed.

To illustrate the issue, we start by recalling the relationship between the Ricci and the Riemann tensors [9]. This is defined as follows:

$R_{\mu \nu}=g^{\alpha \beta} R_{\alpha \mu \beta \nu} \equiv R_{\mu \alpha \nu}^{\alpha}$.

The Riemann tensor $R_{\alpha \mu \beta v}$ satisfies the symmetry properties:

$R_{\alpha \mu \beta \nu}=-R_{\mu \alpha \beta \nu}=-R_{\alpha \mu \nu \beta}$,

$R_{\alpha \mu \beta \nu}=R_{\beta v \alpha \mu}$

which means that it is antisymmetric with respect to each couple of indices $\alpha, \mu$ and $\beta, v$, while it is symmetric with respect to the permutation of the two couples of indices together. Hence, all diagonal components of $R_{\alpha \mu \beta \nu}$ for each couple of indices $\alpha, \mu$ and $\beta, v$ are zero.

In order to replace the Ricci tensor with the Riemann tensor we must specify the character of their contribution in the Hamiltonian density. In fact, in the synchronous variational principle associated with the action integral (17) we implicitly assumed that $\widehat{R}_{\mu \nu}=\widehat{g}^{\alpha \beta} \widehat{R}_{\alpha \mu \beta \nu}$. This means that the Ricci tensor was a prescribed tensor, since it was a function of prescribed metric tensor and Riemann tensor. Now, in order to include the Riemann tensor but preserve at the same time the functional form of the Hamiltonian density in terms of the Ricci 4-scalar, we must assume the following relationship to hold:

$R_{\mu \nu}=g^{\alpha \beta} \widehat{R}_{\alpha \mu \beta \nu}$.

This means that now the Ricci tensor is no longer assumed as an independent prescribed tensor belonging to the background solution, but it is a composite tensor depending on the variational tensor $g^{\alpha \beta}$ and the background Riemann tensor $\widehat{R}_{\alpha \mu \beta \nu}$. This distinction allows one to cast the synchronous variational principle in terms of the Riemann tensor coupling as required for the scope of the present study.

Since the contribution of the Riemann tensor enters in the potential part of the Hamiltonian function, we can focus initially our attention on determining the correct form of the Hamiltonian potential alone. The solution to the question is not unique. For example, a first possibility could be to search for a 4-scalar potential of the form
$V_{G w}(g, \widehat{x}) \equiv \kappa w\left[g^{\mu \nu} g^{\alpha \beta} \widehat{R}_{\alpha \mu \beta \nu}-2 \Lambda\right]$.

This expression follows from Eq. (12) by replacing the Ricci tensor with its definition by the Riemann tensor. In order to account for the new quadratic functional dependence of the potential on the variational tensor $g^{\mu \nu}$, the variational function $h$ is replaced here with the new 4-scalar function $w$. The latter must then be constructed in such a way to warrant the recovery of the extremal equations in terms of EFE. A direct calculation yields

$w \equiv\left(\frac{3}{2}-\frac{1}{4} g_{i k} g^{i k}\right)$

We notice however that by construction $w=h-\frac{1}{2}$, so that the two variational functions $w$ and $h$ remain effectively distinguished in this approach and therefore would generate corresponding different variational forms. In addition, we have that also the extremal values of these functions are different, since $h(\widehat{g})=1$ while $w(\widehat{g})=\frac{1}{2}$. As a consequence, the choice (35) appears against the physical meaning of the variational function $h$ (which, as recalled above, establishes the relationship between synchronous and asynchronous principles). Furthermore, the adoption of $w$ in place of $h$ for the gravitational potential $V_{G w}(g, \widehat{x})$ would place a difference between the form of the gravitational part of the potential, namely $V_{G w}(g, \widehat{x})$, and the external-field potential $V_{F}(g, \widehat{x}, r)$ which instead carries always the function $h$. These features suggest the inadequacy of the representation (34) and the necessity to look for another solution of the problem.

In order to resolve the indeterminacy at classical level and obtain the appropriate form of the classical Riemanncoupling solution that is suitable for the subsequent study of the quantum coupling and for a comparison with the Riccitensor solution, we introduce the following requirement. Namely, starting from the representation of the potential expressed in terms of the Riemann tensor (i.e., the Riemanntensor potential, denoted here $V_{G *}(g, \widehat{x})$ to distinguish it from $\left.V_{G w}(g, \widehat{x})\right)$, it should be possible to recover the form of the corresponding Ricci-tensor potential $V_{G h}(g, \widehat{x})$ given by Eq. (12) by means of the replacement/identification

$g^{\mu \nu} g^{\alpha \beta} \widehat{R}_{\alpha \mu \beta \nu} \rightarrow g^{\mu \nu} \widehat{g}^{\alpha \beta} \widehat{R}_{\alpha \mu \beta \nu} \equiv g^{\mu \nu} \widehat{R}_{\mu \nu}$

in the Hamiltonian. This means that, a posteriori, it should always be possible to relate via Eq. (36) the two Riemanntensor and Ricci-tensor representations. In addition, the Frechet derivative of the corresponding Riemann-tensor potential $V_{G *}(g, \widehat{x})$ should coincide identically with the Frechet derivative of $V_{G h}(g, \widehat{x})$, namely

$\delta V_{G *}(g, \widehat{x}) \equiv \delta V_{G h}(g, \widehat{x})$, 
or equivalent

$$
\left.\left.\frac{d}{d \lambda} V_{G *}(g+\lambda \delta g, \widehat{x})\right|_{\lambda=0} \equiv \frac{d}{d \lambda} \delta V_{G h}(g+\lambda \delta g, \widehat{x})\right|_{\lambda=0},
$$

so that both potentials should recover exactly EFE. The implication of Eq. (37) is therefore that the two potentials $V_{G *}(g, \widehat{x})$ and $V_{G h}(g, \widehat{x})$ should only differ by some suitable gauge function (which, as such, therefore do not contribute to the final Euler-Lagrange equations).

One can see at once that this criterion is sufficient to exclude the validity of the potential (34) in the present framework. In fact, if we promote $g^{\alpha \beta} \rightarrow \widehat{g}^{\alpha \beta}$ when it appears in the coupling with the Riemann tensor in Eq. (34), we would obtain for $V_{G w}(g, \widehat{x})$ in such a limit

$$
\begin{aligned}
& V_{G w}(g, \widehat{x}) \equiv \kappa\left(\frac{3}{2}-\frac{1}{4} g_{i k} g^{i k}\right)\left[g^{\mu \nu} \widehat{R}_{\mu \nu}-2 \Lambda\right] \\
& =V_{G h}(g, \widehat{x})-\frac{1}{2} \kappa\left[g^{\mu \nu} \widehat{R}_{\mu \nu}-2 \Lambda\right] \neq V_{G h}(g, \widehat{x}) .
\end{aligned}
$$

However, one can show that a possible alternate realization of the potential which actually fulfills the requirement is provided by the following non-trivial representation:

$V_{G *}(g, \widehat{x}) \equiv \kappa h\left[g^{\mu \nu} g^{\alpha \beta} \widehat{R}_{\alpha \mu \beta \nu}-2 \Lambda\right]-\kappa g^{\mu \nu}\left(g^{\alpha \beta}-\widehat{g}^{\alpha \beta}\right) \widehat{R}_{\alpha \mu \beta \nu}$,

where $h$ preserves its original form given by Eq. (14). In this case it is immediate to verify that $V_{G *}(g, \widehat{x})$ is related to $V_{G h}(g, \widehat{x})$ by means of the transformation (36). Indeed, when letting $g^{\alpha \beta} \rightarrow \widehat{g}^{\alpha \beta}$ both in the square and round bracket terms in Eq. (39) one obtains that

$\lim _{g^{\alpha \beta} \rightarrow \widehat{g}^{\alpha \beta}} V_{G *}(g, \widehat{x})=V_{G h}(g, \widehat{x})$.

Furthermore, one notices that by construction

$V_{G *}(g, \widehat{x})=V_{G h}(g, \widehat{x})+F_{G *}(g, \widehat{x})$,

where

$F_{G *}(g, \widehat{x}) \equiv \kappa h\left[g^{\mu \nu} g^{\alpha \beta} \widehat{R}_{\alpha \mu \beta \nu}-g^{\mu \nu} \widehat{R}_{\mu \nu}\right]-\kappa g^{\mu \nu}\left(g^{\alpha \beta}-\widehat{g}^{\alpha \beta}\right) \widehat{R}_{\alpha \mu \beta \nu}$.

Now, one can prove by straightforward algebra that identically

$\delta F_{G *}(\widehat{g}, \widehat{x})=0$

holds, so that $F_{G *}(g, \widehat{x})$ indeed identifies a gauge function. Consequently Eq. (37) applies too.
We now notice that the potential $V_{G *}(g, \widehat{x})$ is made of two contributions, both carrying a coupling with the background Riemann tensor. The first one is proportional to $h$ and measures how the product $g^{i k} g_{i k}$ differs from its normalized value, namely $\widehat{g}^{i k} \widehat{g}_{i k}$. The second term is proportional to the difference $\left(g^{\alpha \beta}-\widehat{g}^{\alpha \beta}\right)$ which measures the strength of the coupling with Riemann tensor by the variational field $g^{\alpha \beta}$ with respect to the same coupling realized by the background tensor $\widehat{g}^{\alpha \beta}$.

Given these premises, we can now identify the Hamiltonian function as $H \equiv H_{*}$, where $H_{*} \equiv H_{*}(x, \widehat{x}, r, s)$ is defined as follows:

$H_{*}=T-V_{*}$,

where the effective kinetic and potential densities $T \equiv T(x, \widehat{x})$ and $V_{*} \equiv V_{*}(g, \widehat{x}, r, s)$ are of the form

$$
\begin{aligned}
T(x, \widehat{x}) & \equiv \frac{1}{2 \kappa} \pi_{\mu \nu} \pi^{\mu \nu}, \\
V_{*}(g, \widehat{x}, r, s) & \equiv V_{G *}(g, \widehat{x})+V_{F}(g, \widehat{x}, r, s) .
\end{aligned}
$$

We notice that the external potential $V_{F}(g, \widehat{x}, r, s)$ has here the same representation given by Eq. (13) in terms of the function $h$ given by Eq. (14). Then, the difference between $H_{h}$ and $H_{*}$ is only in the prescription of the potential $V_{G *}(g, \widehat{x})$, to be identified with the representation (40) given above. Hence, the synchronous Hamilton variational principle in the case of Riemann tensor dependence is realized by the 4-scalar variational functional

$S_{w}(x, \widehat{x}) \equiv \int d \Omega L_{*}(x, \widehat{x}, r, s)$,

with $d \Omega \equiv d^{4} r \sqrt{-|\widehat{g}|}$ identifying again the invariant 4volume element of the space-time, while $L_{*}(x, \widehat{x}, r, s)$ is the variational Lagrangian density

$L_{*}(x, \widehat{x}, r, s) \equiv \pi_{\mu \nu} \frac{d}{d s} g^{\mu \nu}-H_{*}(x, \widehat{x}, r, s)$,

which identifies the Legendre transform of the corresponding variational Hamiltonian density $H_{*}(x, \widehat{x}, r, s)$ defined by Eq. (45).

The corresponding synchronous variational principle is realized as

$\delta S_{*}(x, \widehat{x})=0$,

which yields the 4-tensor Euler-Lagrange equations

$\left\{\begin{array}{l}\frac{\delta S_{*}(x, \widehat{x})}{\delta g^{\mu v}}=0 \\ \frac{\delta S_{*}(x, \widehat{x})}{\delta \pi_{\mu \nu}}=0\end{array}\right.$ 
that are equivalent to the set of Hamilton equations (3) when $H$ is identified with $H_{*}$.

The explicit calculation of the potential term $V_{G *}(g, \widehat{x})$ carrying the Riemann contribution gives

$$
\begin{aligned}
& \frac{\delta V_{G *}(g, \widehat{x})}{\delta g^{\mu \nu}}=\kappa\left[-\frac{1}{2} g_{\mu \nu}\left(g^{i k} g^{\alpha \beta} \widehat{R}_{\alpha i \beta k}-2 \Lambda\right)\right. \\
& \left.\quad+2 h g^{\alpha \beta} \widehat{R}_{\alpha \mu \beta \nu}-\left(g^{\alpha \beta}-\widehat{g}^{\alpha \beta}\right) \widehat{R}_{\alpha \mu \beta \nu}-g^{\alpha \beta} \widehat{R}_{\alpha \mu \beta \nu}\right] .
\end{aligned}
$$

As a result, the canonical equations (3) yield the following Lagrangian evolution equation for the variational field $g_{\mu \nu}(s)$ :

$$
\begin{aligned}
& \frac{d}{d s}\left[\frac{d}{d s} g_{\mu \nu}(s)\right]-\frac{1}{2} g_{\mu \nu}\left(g^{i k} g^{\alpha \beta} \widehat{R}_{\alpha i \beta k}-2 \Lambda\right) \\
& -\left(2(1-h) g^{\alpha \beta}-\widehat{g}^{\alpha \beta}\right) \widehat{R}_{\alpha \mu \beta \nu}-\frac{8 \pi G}{c^{2}} T_{\mu \nu}=0 .
\end{aligned}
$$

The connection with EFE can then be established in the same way as done for the Ricci tensor case, namely by setting stationary initial conditions of the type (27), and recalling that by definition $\widehat{g}^{\alpha \beta}(s) \widehat{R}_{\alpha \mu \beta \nu}=\widehat{R}_{\mu \nu}$ and $h(\widehat{g})=1$. As a consequence, the correct form of EFE given by Eq. (29) is recovered.

We recall that the asynchronous variational approach to EFE that is customarily implemented in the literature prevents the possibility of expressing the variational Lagrangian density in terms of the Riemann tensor. As a consequence, only Lagrangian densities in which the Ricci scalar is represented in terms of the Ricci tensor are admitted for the correct variational derivation of EFE. However, the theory reported here proves that this obstacle can be consistently overcome in the framework of synchronous variational principles. In particular, the requirement of satisfying the principle of manifest covariance and the possibility of implementing a background formalism provide the necessary freedom to handle the problem. In this way, the synchronous variational approach is shown to permit the construction of 4-scalar Lagrangian and Hamiltonian densities in which the gravitational potential term can be expressed either in terms of the Ricci tensor or the Riemann tensor. Both representations determine the correct form of EFE and are related to each other according to the criterion given above.

We have therefore succeeded in providing two different but equivalent classical variational Hamiltonian formulations for EFE, which display the contributions of Ricci and Riemann tensors. In both approaches these two curvature tensors enter as prescribed background quantities, which are therefore to be determined a posteriori as consistent solutions of the extremal field equations, namely EFE. In the classical variational framework the two tensors are both coupled with the variational field tensor $g^{\mu \nu}(s)$, but in different manner. As shown below, this coupling is then inherited by the quantum gravitational field. In particular, we stress that in the realm of classical theory in principle both solutions are equally admissible, since the difference pertains the variational (i.e., virtual) fields, while the extremal equations coincide in both cases with EFE for a given initial condition on the canonical state. This proves the convenience of adopting synchronous variational principles instead of asynchronous ones. However, when passing to the quantum domain by means of canonical quantization of the classical Hamiltonian structure, the corresponding quantum Hamiltonian operators give rise to different physical dynamics for the quantum field (which recovers the variational field in the semiclassical limit). As shown below, the selection of the correct coupling solution between Ricci or Riemann tensors can be established based on quantum gravity theory.

\section{Stationary eigenvalue quantum-gravity wave equation}

The classical synchronous variational principles expressed in terms of manifestly-covariant Hamiltonian densities generate a corresponding quantum theory of the gravitational field. The mathematical framework is provided by the manifestlycovariant quantum gravity theory (CQG-theory) developed in Refs. [1,2], which satisfies the requirement 3 set in the Introduction. When the dimensional analysis is taken into account, the mapping (6) requires specifically that $\pi_{\mu \nu} \rightarrow$ $\frac{\kappa}{\alpha L} \pi_{\mu \nu}^{(q)}$ and $H \rightarrow \frac{\kappa}{\alpha L} H^{(q)}$, where $\alpha L$ is a suitable dimensional constant related to the graviton mass and the cosmological constant, determined in Ref. [2].

In this context, the quantum-wave equation takes the form of the Schrödinger-like equation (7) for the 4-scalar wave function $\psi(s) \equiv \psi(g, \widehat{g}, r, s)$, where the evolution parameter is represented by the invariant proper-time $s$. This wave equation admits generally non-stationary solutions for $\psi(s)$ in which the proper-time dependence cannot be simply factored out. This occurrence is an intrinsic feature of CQGtheory, and it follows because the CQG wave equation admits generally solutions which are far from the classical one, i.e., the prescribed background solution $\widehat{g}_{\mu \nu}(r)$, which is instead stationary by assumption.

However, for the purpose of the present study, we need to evaluate the stationary eigenvalue form of the CQG-wave equation in order to question whether the Riemann and Ricci Hamiltonian potentials are both admissible. In particular, in order to represent physical solutions, we require that both potentials should define a positive-definite quadratic form upon performing a quadratic expansion around equilibrium. In the framework of CQG-theory, this requirement warrants the existence of positive eigenvalues for the stationary spectrum of the quantum Hamiltonian and hence predicts in turn a strictly-positive graviton mass. 
Hence, for definiteness we assume that the Hamiltonian operator $H^{(q)}$ obtained by the quantum correspondence principle from the classical potentials $H_{h}$ or $H_{*}$ defined above does not depend on $s$, at least in an asymptotic sense. It follows that $\psi(s)$ must admit a separable particular solution of the form

$\psi(s)=\exp \left\{-\frac{i}{\hbar c} E\left(s-s_{o}\right)\right\} \psi_{o}(g, \widehat{g}, r)$,

where $E$ is a real constant 4-scalar independent of the proper time $s$. As a result, $E$ is the solution of the proper-time dependent equation

$i \hbar \frac{\partial}{\partial s} \psi(s)=\frac{E}{c} \psi(s)$.

Then, $\psi_{o}(g) \equiv \psi_{o}(g, \widehat{g}, r)$ is necessarily a solution of the asymptotic proper-time independent quantum wave equation

$H^{(q)} \psi_{o}(g)=\frac{E}{c} \psi_{o}(g)$,

to be referred to as stationary eigenvalue CQG-wave equation.

In order to proceed investigating the issue on quantum coupling with Ricci and Riemann tensors we need to determine the analytical representation of the eigenvalue equation (55) for the two cases in which $H^{(q)}=H_{h}$ and $H^{(q)}=H_{*}$ respectively. We therefore introduce below the formalism required for this task in terms of an appropriate perturbative approximation scheme. To start with, we consider for the quantum field $g_{\mu \nu}$ a decomposition of the form

$g_{\mu \nu}=\widehat{g}_{\mu \nu}(r)+\delta g_{\mu \nu}$,

with $\delta g^{\mu \nu}$ being the quantum displacement 4-tensor field, to be assumed suitably small with respect to $\widehat{g}_{\mu \nu}(r)$. Hence, $g_{\mu \nu}$ is required to belong to a suitable infinitesimal neighborhood of $\widehat{g}(r) \equiv\left\{\widehat{g}_{\mu \nu}(r)\right\}$, i.e., the subset $U_{g}$ defined as

$U_{g}(\widehat{g}(r), \varepsilon)=\left\{g_{\mu \nu} \equiv \widehat{g}_{\mu \nu}(r)+\delta g_{\mu \nu}, \delta g_{\mu \nu}\right.$

$$
\left.\lesssim O(\varepsilon), g_{\mu \nu} \in U_{g}\right\} \text {, }
$$

such that for all displacements $\delta g_{\mu \nu}$ the asymptotic ordering

$\delta g_{\mu \nu} \lesssim O(\varepsilon)$

holds. Here $\varepsilon$ is a suitable infinitesimal real parameter, while by construction in such a set all components of $\delta g_{\mu \nu}$ are of $O(\varepsilon)$ or higher. The consequence of this assumption is that $\psi_{o}(g, \widehat{g}, r)$ must be regarded suitably localized in the neighborhood of the background equilibrium solution $\widehat{g}(r) \equiv\left\{\widehat{g}_{\mu \nu}(r)\right\}$ so that possible additional classical stationary solutions can be effectively ignored.
In addition to the decomposition (56), we must also prescribe the form of the background metric tensor $\widehat{g}_{\mu \nu}(r)$. Here we assume for definiteness that $V_{F}=0$, so that this is identified with the vacuum deSitter solution of EFE in the presence of a non-vanishing cosmological constant $\Lambda>0$. This represents in fact the simplest curved space-time solution characterized by absence of external fields other than the gravitational one, and having a constant invariant curvature generated by $\Lambda$, so that both $\widehat{R}_{\mu \nu} \neq 0$ and $\widehat{R}_{\alpha \mu \beta \nu} \neq 0$. More precisely, the deSitter solution yields analytical representations for the background curvature tensors in terms of the metric tensor, given by

$$
\begin{aligned}
\widehat{R} & =4 \Lambda, \\
\widehat{R}_{\mu \nu} & =\Lambda \widehat{g}_{\mu \nu}, \\
\widehat{R}_{\alpha \mu \beta \nu} & =\frac{\Lambda}{3}\left(\widehat{g}_{\alpha \beta} \widehat{g}_{\mu \nu}-\widehat{g}_{\alpha \nu} \widehat{g}_{\mu \beta}\right) .
\end{aligned}
$$

Given these premises, the strategy to be pursued is as follows:

(1) We consider the two quantum Hamiltonian solutions corresponding to $H^{(q)}=H_{h}$ and $H^{(q)}=H_{*}$ respectively. For each of them we introduce the vacuum assumption and then perturb the Hamiltonian potential of the gravitational field by introducing the decomposition (56).

(2) We then assume to have a background deSitter solution of EFE and we truncate the perturbative expression of the potential to second order in the displacement $\delta g_{\mu \nu}$, letting the zeroth and first order terms to vanish identically in a local minimum of the potential.

(3) We finally analyze whether the remaining terms of the second-order Hamiltonian potential can admit a discrete value of eigenvalues, to coincide with the fundamental quantum harmonic-oscillator solution. This in turn demands the possibility of implementing the Dirac ladder method to the eigenvalue equation. For the validity of this approach, however, the Hamiltonian potential must represent a quadratic and positive-definite form, in order to warrant existence of corresponding positive eigenvalues.

(4) Depending on the resulting outcome of this analytical analysis, it is concluded whether the two quantum configurations $H^{(q)}=H_{h}$ and $H^{(q)}=H_{*}$ can both represent quantum gravitational descriptions in the deSitter background spacetime. In particular, this permits to answer the question about the physical connotation and admissible character of the couplings of the quantum gravitational field with the Ricci and Riemann curvature tensors.

\section{Quantum potentials with Ricci and Riemann tensors}

In this section we consider the application of the asymptotic approximation scheme reported above, to be implemented on 
the Hamiltonian potentials of the stationary eigenvalue CQGwave equation expressed respectively in terms of Ricci and Riemann tensors.

More precisely, in the case of the Ricci tensor we investigate the analytical expression of the potential $V_{G h} \equiv$ $V_{G h}(g, \widehat{x})$ given by Eq. (12), which is written explicitly as

$$
V_{G h} \equiv \kappa\left(2-\frac{1}{4} g^{i k} g_{i k}\right)\left[g^{\mu \nu} \widehat{R}_{\mu \nu}-2 \Lambda\right]
$$

Then, introducing the decomposition (56) one obtains

$V_{G h} \equiv \kappa\left(2-\frac{1}{4}\left(\widehat{g}^{i k}+\delta g^{i k}\right)\left(\widehat{g}_{i k}+\delta g_{i k}\right)\right)\left[\left(\widehat{g}^{\mu \nu}+\delta g^{\mu \nu}\right) \widehat{R}_{\mu \nu}-2 \Lambda\right]$

Recalling the definition of the Ricci tensor and making use of the normalization $\widehat{g}^{i k} \widehat{g}_{i k}=4$ gives

$$
V_{G h}=\kappa\left(1-\frac{1}{2} \delta g^{i k} \widehat{g}_{i k}-\frac{1}{4} \delta g^{i k} \delta g_{i k}\right)\left[\delta g^{\mu \nu} \widehat{R}_{\mu \nu}+\widehat{R}-2 \Lambda\right]
$$

Assuming to be in a local minimum of the potential, the equation associated with the linear terms is identically satisfied. It is immediate to verify that this is the classical EFE, which is consistent with the decomposition (56) performed around the stationary background metric tensor $\widehat{g}_{\mu \nu}$. Hence, in Eq. (64) it is meaningful to retain only the quadratic terms of the expansion. This yields the approximate expression

$V_{G h}=U_{G h}+O\left(\varepsilon^{3}\right)$

where the leading-order contribution $U_{G h}$ is found to be

$U_{G h}=-\kappa \frac{1}{2} \delta g^{i k} \widehat{g}_{i k}\left(\delta g^{\mu \nu} \widehat{R}_{\mu \nu}\right)-\kappa \frac{1}{4} \delta g^{i k} \delta g_{i k}(\widehat{R}-2 \Lambda)$.

Then, for a deSitter background solution, invoking the relations (59) and (60) gives

$U_{G h}=-\kappa \Lambda \frac{1}{2} \delta g^{i k} \widehat{g}_{i k} \delta g^{\mu \nu} \widehat{g}_{\mu \nu}-\kappa \Lambda \frac{1}{2} \delta g^{i k} \delta g_{i k}$.

Introducing the notation $\delta g^{2}=\delta g^{\mu \nu} \delta g_{\mu \nu}$ we can finally write the approximate potential as

$U_{G h}=-\kappa \Lambda \frac{1}{2}\left[\left(\delta g^{\mu \nu} \widehat{g}_{\mu \nu}\right)^{2}+\delta g^{2}\right]$

This result recovers the solution originally obtained in Ref. [2]. We notice that for the deSitter space-time, $U_{G h}$ defines a positive-definite quadratic form. This expression is therefore consistent with the implementation of the Dirac ladder method for the determination of the discrete eigenvalue spectrum associated with Eq. (55). In fact, it formally recovers the functional form of the quantum potential driving a quantum harmonic oscillator.

Let us now consider instead the analogous algebraic problem associated with the potential $V_{G *} \equiv V_{G *}(g, \widehat{x})$ defined in Eq. (40), which is written explicitly as

$$
\begin{aligned}
& V_{G *} \equiv \kappa\left(2-\frac{1}{4} g^{i k} g_{i k}\right)\left[g^{\mu \nu} g^{\alpha \beta} \widehat{R}_{\alpha \mu \beta \nu}-2 \Lambda\right] \\
& -\kappa g^{\mu \nu}\left(g^{\alpha \beta}-\widehat{g}^{\alpha \beta}\right) \widehat{R}_{\alpha \mu \beta \nu} .
\end{aligned}
$$

Introducing the linear decomposition (56) one obtains

$$
\begin{aligned}
V_{G *}= & \kappa\left(2-\frac{1}{4}\left(\widehat{g}^{i k}+\delta g^{i k}\right)\left(\widehat{g}_{i k}+\delta g_{i k}\right)\right) \\
& \times\left[\left(\widehat{g}^{\mu \nu}+\delta g^{\mu \nu}\right)\left(\widehat{g}^{\alpha \beta}+\delta g^{\alpha \beta}\right) \widehat{R}_{\alpha \mu \beta \nu}-2 \Lambda\right] \\
& -\kappa\left(\widehat{g}^{\mu \nu}+\delta g^{\mu \nu}\right)\left(\widehat{g}^{\alpha \beta}+\delta g^{\alpha \beta}-\widehat{g}^{\alpha \beta}\right) \widehat{R}_{\alpha \mu \beta \nu} .
\end{aligned}
$$

Using now the definitions of the Ricci and Riemann tensors and the normalization $\widehat{g}^{i k} \widehat{g}_{i k}=4$ gives

$$
\begin{aligned}
V_{G *}= & \kappa\left(1-\frac{1}{2} \delta g^{i k} \widehat{g}_{i k}-\frac{1}{4} \delta g^{i k} \delta g_{i k}\right) \\
& \times\left[\left(\delta g^{\alpha \beta} \widehat{g}^{\mu \nu}+\delta g^{\mu v} \delta g^{\alpha \beta}\right) \widehat{R}_{\alpha \mu \beta \nu}+\delta g^{\mu \nu} \widehat{R}_{\mu \nu}+\widehat{R}-2 \Lambda\right] \\
& -\kappa\left(\widehat{g}^{\mu \nu} \delta g^{\alpha \beta}+\delta g^{\mu \nu} \delta g^{\alpha \beta}\right) \widehat{R}_{\alpha \mu \beta \nu} .
\end{aligned}
$$

Again one can verify that the equation associated with the linear terms is identically satisfied and coincides with EFE. Hence, the quadratic terms of the expansion in Eq. (71) yield the following approximate expression

$V_{G *}=U_{G *}+O\left(\varepsilon^{3}\right)$

where the leading-order contribution $U_{G *}$ is given by

$$
\begin{aligned}
U_{G *}= & -\kappa\left[\delta g^{\mu \nu} \delta g^{\alpha \beta} \widehat{R}_{\alpha \mu \beta \nu}\right] \\
& -\kappa \frac{1}{2} \delta g^{i k} \widehat{g}_{i k}\left[\delta g^{\alpha \beta} \widehat{g}^{\mu \nu} \widehat{R}_{\alpha \mu \beta \nu}+\delta g^{\mu \nu} \widehat{R}_{\mu \nu}\right] \\
& -\kappa \frac{1}{4} \delta g^{i k} \delta g_{i k}[\widehat{R}-2 \Lambda]-\kappa \delta g^{\mu v} \delta g^{\alpha \beta} \widehat{R}_{\alpha \mu \beta \nu} .
\end{aligned}
$$

Substituting the relations (59)-(61) applying for a deSitter background solution and simplifying the expression by introducing the notation $\delta g^{2}=\delta g^{\mu \nu} \delta g_{\mu \nu}$ we then get

$$
\begin{aligned}
U_{G *}= & -\kappa \frac{\Lambda}{3} \delta g^{\mu \nu} \widehat{g}_{\mu \nu} \delta g^{\alpha \beta} \widehat{g}_{\alpha \beta}+\kappa \Lambda \frac{1}{6} \delta g^{2} \\
& -\kappa \Lambda \frac{1}{2} \delta g^{i k} \widehat{g}_{i k}\left[\frac{4}{3} \delta g^{\alpha \beta} \widehat{g}_{\alpha \beta}-\frac{1}{3} \delta g^{\mu \nu} \widehat{g}_{\mu \nu}+\delta g^{\mu \nu} \widehat{g}_{\mu \nu}\right] \\
& -\kappa \frac{\Lambda}{3} \delta g^{\mu \nu} \widehat{g}_{\mu \nu} \delta g^{\alpha \beta} \widehat{g}_{\alpha \beta},
\end{aligned}
$$

which reduces to

$U_{G *}=-\kappa \Lambda \frac{5}{3} \delta g^{\mu \nu} \widehat{g}_{\mu \nu} \delta g^{\alpha \beta} \widehat{g}_{\alpha \beta}+\kappa \Lambda \frac{1}{6} \delta g^{2}$. 
Finally, for a comparison with Eq. (68) we write the previous expression in the form

$U_{G *}=\kappa \Lambda\left[\frac{1}{6} \delta g^{2}-\frac{5}{3}\left(\delta g^{\mu \nu} \widehat{g}_{\mu \nu}\right)^{2}\right]$.

This expression however does not represent a positivedefinite quadratic form since the sign of the difference between $\delta g^{2}$ and $\left(\delta g^{\mu \nu} \widehat{g}_{\mu \nu}\right)^{2}$ cannot be established a priori.

It is interesting to point out that the reason for this failure is due to the anti-symmetry properties of the Riemann tensor. In the vacuum case for the cosmological deSitter solution in fact the analytical representation (61) applies. Thus, the coupling of the quantum gravitational field with the Riemann tensor generates the corresponding difference between the squared of the modulus of $\delta g^{\mu \nu}$ and the squared of its trace. In contrast, the coupling with the Ricci tensor displayed above is not affected by this antisymmetric property, and remains therefore consistent with the requirements for constructing the quantum harmonic solution.

\section{Discussion and concluding remarks}

From the conceptual point of view, the possibility of retaining different dynamical couplings of the quantum gravitational field, i.e., respectively both with the Riemann and Ricci curvature tensors, is in principle admissible. In this paper the issue has been addressed in order to elucidate the mathematical properties of such couplings and the physical reasons behind the validity of either possible realizations. In particular, the analysis of the coupling of the gravitational field with either Riemann or Ricci tensors requires, at classical level, setting the problem by starting with the appropriate prescriptions for the Hamilton variational principles in the two cases. To reach the target requires setting the problem in the framework of classical synchronous variational principles and the corresponding realization of manifestly-covariant quantum gravity theory. Accordingly, the identification of the classical Hamiltonian structure underlying the Einstein field equations (EFE) of General Relativity (GR) permits determination of the quantum Hamiltonian structure and related quantum wave equation in terms of standard canonical quantization methods.

Concerning the classical theory, two different realizations of the synchronous variational principle, both yielding EFE, have therefore been proposed, whose Hamiltonian functions express respectively the coupling of the classical variational gravitational tensor with the Ricci and Riemann tensors. It has been shown that, upon imposing a suitable physical requirement, it is in principle possible to select a unique form of the two synchronous classical principles. However, at the classical level, such requirement remains not mandatory in character. Thus, both Ricci- and Riemann-tensor couplings appear admissible, since they recover the same form of EFE as extremal field equations. On the other hand, such an outcome appears of interest because it overcomes what may appear a limitation characteristic of former literature variational approaches based on asynchronous variational principles, like the Einstein-Hilbert one. In such as context, in fact, to achieve EFE only the Ricci-tensor coupling is admissible.

Nevertheless, the indeterminacy feature discovered here represents also a possible issue at the quantum level, because it implies a possible non-uniqueness feature of quantum theory. A possible serious question of consistency therefore arises in the formulation of quantum gravity theory. The possibility of representing two equivalent classical variational principles in terms of either the Ricci or Riemann tensors must therefore be solved in the framework of quantum Hamiltonian theory of GR, i.e., only provided the admissible character of the two possible solutions can be established at the classical level. This means that also quantum theory must be based on a synchronous variational principle. A quantum theory with such a characteristic is indeed the covariant theory of quantum gravity, referred to shortly as CQGtheory. A precise physical requirement has been imposed in this regard. This amounts to warrant that the functional form of the quantum Hamiltonian operator must admit a quantum harmonic oscillator solution in terms of a quadratic and positive-definite Hamiltonian potential, upon performing a quadratic expansion around equilibrium. From the physical point of view, this criterion warrants the existence of a discrete spectrum of positive eigenvalues that is associated with the prediction of existence of quantum gravitons having strictly-positive non-vanishing mass.

However, from the physics standpoint, the above requirement has a deeper meaning. In fact, CQG-theory is the massive-graviton and 4-tensor theory of manifestly-covariant QG which pertains the Einstein Field Equations (EFE) and its related abstract 4-tensor classical Hamiltonian structure. As such, CQG-theory applies only provided it consistently predicts also a strictly positive spectrum of invariant (i.e., rest) masses for gravitons. The conclusion is therefore that the above requirement is nothing less than a consistency requirement for the validity of the whole quantum theory itself.

As discussed in the paper, the mathematical procedure adopted for the task outlined above has consisted in the analysis of the functional form of the quantum Hamiltonian potential for the two solutions separately. For this purpose, a suitable perturbative analysis has been performed, in which the quantum state is expanded around the equilibrium stationary space-time configuration. The latter is represented by the deSitter space-time in the presence of a non-vanishing cosmological constant. The vacuum deSitter geometry in fact represents the simplest configuration characterized by a identified space-time with constant curvature, generating a uni- 
form potential well where the discrete eigenvalue spectrum must be realized.

It has been proved that, contrary to the classical theory, at quantum level the Ricci and Riemann tensor-couplings are not equivalent even if they correspond to different realizations of the vacuum potential which are related via suitable gauge-transformations. In fact, although both the Ricci and Riemann potentials lead to the same Euler-Lagrange equations (i.e., EFE), the asymptotic behavior of the same potentials differ. More precisely, the asymptotic potential $U_{G h}$ containing the Ricci tensor yields a quadratic and positivedefinite form. In contrast, the Riemann tensor coupling occurring in the potential $U_{G *}$ generates a quadratic but nonpositive definite form. This leads to a mandatory conclusion which permits to identify a unique selection criterion for the vacuum coupling-potential. Indeed the physicallymeaningful tensor coupling that leads to an acceptable solution occurs through the Ricci tensor, while the Riemanntensor potential is ruled out. The underlying physical motivation is related to the non-symmetric properties of the Riemann tensor. The conclusion confirms also the correctness of the quantum-gravity solution obtained in Ref. [2] in terms of the Ricci tensor dependence in the Hamiltonian potential. At the same time, the theory developed in this paper can provide a convenient background for the investigation of classical variational and quantum Hamiltonian descriptions of space-time dynamics as well the tensor-coupling existing between quantum gravitational field and the curvature tensors of General Relativity.

Data Availability Statement This manuscript has no associated data or the data will not be deposited. [Authors' comment: This is a theoretical research. All data produced during this study are contained in this published article.]

Open Access This article is licensed under a Creative Commons Attribution 4.0 International License, which permits use, sharing, adaptation, distribution and reproduction in any medium or format, as long as you give appropriate credit to the original author(s) and the source, provide a link to the Creative Commons licence, and indicate if changes were made. The images or other third party material in this article are included in the article's Creative Commons licence, unless indicated otherwise in a credit line to the material. If material is not included in the article's Creative Commons licence and your intended use is not permitted by statutory regulation or exceeds the permitted use, you will need to obtain permission directly from the copyright holder. To view a copy of this licence, visit http://creativecomm ons.org/licenses/by/4.0/.

Funded by SCOAP ${ }^{3}$.

\section{References}

1. C. Cremaschini, M. Tessarotto, Hamiltonian approach to GRpart 1: covariant theory of classical gravity. Eur. Phys. J. C 77, 329 (2017)

2. C. Cremaschini, M. Tessarotto, Hamiltonian approach to GRpart 2: covariant theory of quantum gravity. Eur. Phys. J. C 77, 330 (2017)
3. N. Christiansen, K. Falls, J.M. Pawlowski, M. Reichert, Curvature dependence of quantum gravity. Phys. Rev. D 97, 046007 (2018)

4. M. Mihai, Dynamical aspects for scalar fields coupled to cubic contractions of the Riemann tensor. Phys. Rev. D 102, 023517 (2020)

5. F. Kuipers, X. Calmet, Singularity theorems in the effective field theory for quantum gravity at second order in curvature. Universe 6, $171(2020)$

6. J. Brunekreef, R. Loll, Curvature profiles for quantum gravity. Phys. Rev. D 103, 026019 (2021)

7. F. Kuipers, Stochastic quantization on Lorentzian manifolds. J. High Energy Phys. 2021, 05 (2021)

8. A. Cisterna, A. Neira-Gallegos, J. Oliva, S.C. Rebolledo-Caceres, Plebański-Demiański solutions in quadratic gravity with conformally coupled scalar fields. Phys. Rev. D 103, 064050 (2021)

9. L.D. Landau, E.M. Lifschitz, Field Theory, Theoretical Physics, vol. 2 (Addison-Wesley, New York, 1957)

10. S. Nojiri, S.D. Odintsov, A proposal for covariant renormalizable field theory of gravity. Phys. Lett. B 691, 60 (2010)

11. M. Blaschke, Z. Stuchlík, F. Blaschke, P. Blaschke, Classical corrections to black hole entropy in d dimensions: a rear window to quantum gravity? Phys. Rev. D 96, 104012 (2017)

12. G. Magnano, K.A. Meissner, L.M. Sokołowski, Test-field limit of metric nonlinear gravity theories. Eur. Phys. J. C 79, 631 (2019)

13. A. Einstein, The Meaning of Relativity (Princeton University Press, Princeton, 2004). U.S.A N.J.,

14. M. Tessarotto, C. Cremaschini, The principle of covariance and the Hamiltonian formulation of general relativity. Entropy 23, 215 (2021)

15. F. Moulin, Generalization of Einstein's gravitational field equations. Eur. Phys. J. C 77, 878 (2017)

16. C. Cremaschini, M. Tessarotto, Synchronous Lagrangian variational principles in general relativity. Eur. Phys. J. Plus 130, 123 (2015)

17. E.I. Guendelman, A.B. Kaganovich, The principle of nongravitating vacuum energy and some of its consequences. Phys. Rev. D 53, 7020 (1996)

18. E.I. Guendelman, Scale invariance, new inflation and decaying $\Lambda$ terms. Mod. Phys. Lett. A 14, 1043 (1999)

19. D. Benisty, E.I. Guendelman, E. Nissimov, S. Pacheva, Dynamically generated inflation from non-Riemannian volume forms. Eur. Phys. J. C 79, 806 (2019)

20. C. Cremaschini, M. Tessarotto, Space-time second-quantization effects and the quantum origin of cosmological constant in covariant quantum gravity. Symmetry 10, 287 (2018)

21. J. Struckmeier, A. Redelbach, Covariant Hamiltonian field theory. Int. J. Mod. Phys. E 17, 435 (2008)

22. R. Arnowitt, S. Deser, C.W. Misner, in Gravitation: An Introduction to Current Research, ed. by L. Witten (Wiley, New York, 1962)

23. A. Ashtekar, Gravity and the quantum. New J. Phys. 7, 198 (2005)

24. C. Rovelli, L. Smolin, Loop space representation of quantum general relativity. Nucl. Phys. B 331, 80 (1990)

25. A. Messiah, Quantum Mechanics (Dover Publications, New York, 1999) 